\title{
SUR LA DETERMINATION DES FEMELLES DE POTAMOPHYLAX LATIPENNNIS (CURT.) NEBOISS ET POTAMOPHYLAX CINGULATUS (STEPHENS) [Trichoptera]
}

\author{
par H. DÉCamps.
}

A la suite de la révision des types de la collection J. Curris [A. Neborss 1963], les deux espèces de Potamophylax couramment récoltées en Europe et figurées dans les travaux fondamentaux de R. Maclachlan [1874] et M. E. Mosely [1939], ont dû être renommées. La nomenclature proposée par A. Nerorss, s'établit ainsi :

- Potamophylax latipennis (Curt.) Neborss = P. stellatus $($ CunT. $)=P$. stellatus auct.

- Potamophylax cingulatus (Stephens) $=P$. latipennis auct.

C'est celle qui est utilisée dans cette note concernant la distinction des deux espèces, du point de vue morphologique et écologique.

\section{1. - Caractères des femelles de $P$. latipennis et $P$. cingulatus.}

1.1. - La distinction des femelles de P. latipennis et $P$. cingulatus est restée inconnue, jusqu'à la publication d'une détermination, basée sur les caractères externes et internes de l'appareil génital $q$ [D. E. Kimmins 1944]. Les critères utilisés portaient sur :

- la partie ventrale du segment IX, plus large chez $P$. latipennis;

- le lobe médian de l'écaille vulvaire, pointu chez $P$. latipennis, émoussé chez $P$. cingulatus;

- des structures différentes de l'appareil vaginal interne.

Par la suite, C. Tomaszewski [1962] ajoutait des observations sur la nervation et la coloration des ailes antérieures de $P$. cingulatus.

1.2. - L'utilisation de ces caractères dans la détermination des exemplaires pyrénéens conduit aux remarques suivantes :

a) Les caractères externes - ailes antérieures, partie ventrale de segment IX, lobe médian de l'écaille vulvaire - sont trop variables pour permettre une séparation constante des deux espèces (fig. 1 à 6 ).

b) la structure interne de l'appareil vaginal distingue plus sûrement $P$. latipennis de $P$. cingulatus et il est possible de préciser ces différences, d'après les données de F. Schmid [1955], sur les appareils génitaux $\&$ des Limnephilides : 
- l'ouverture du vestibule vaginal se poursuit par une partie rétrécie qui, chez $P$. latipennis, présente latéralement deux prolongements en pointe situés au-dessus de la pièce centrale (fig. 7). Chez $P$. cingulatus, cette partie est plus étroite et ne possède pas de prolongements latéraux (fig. 8).
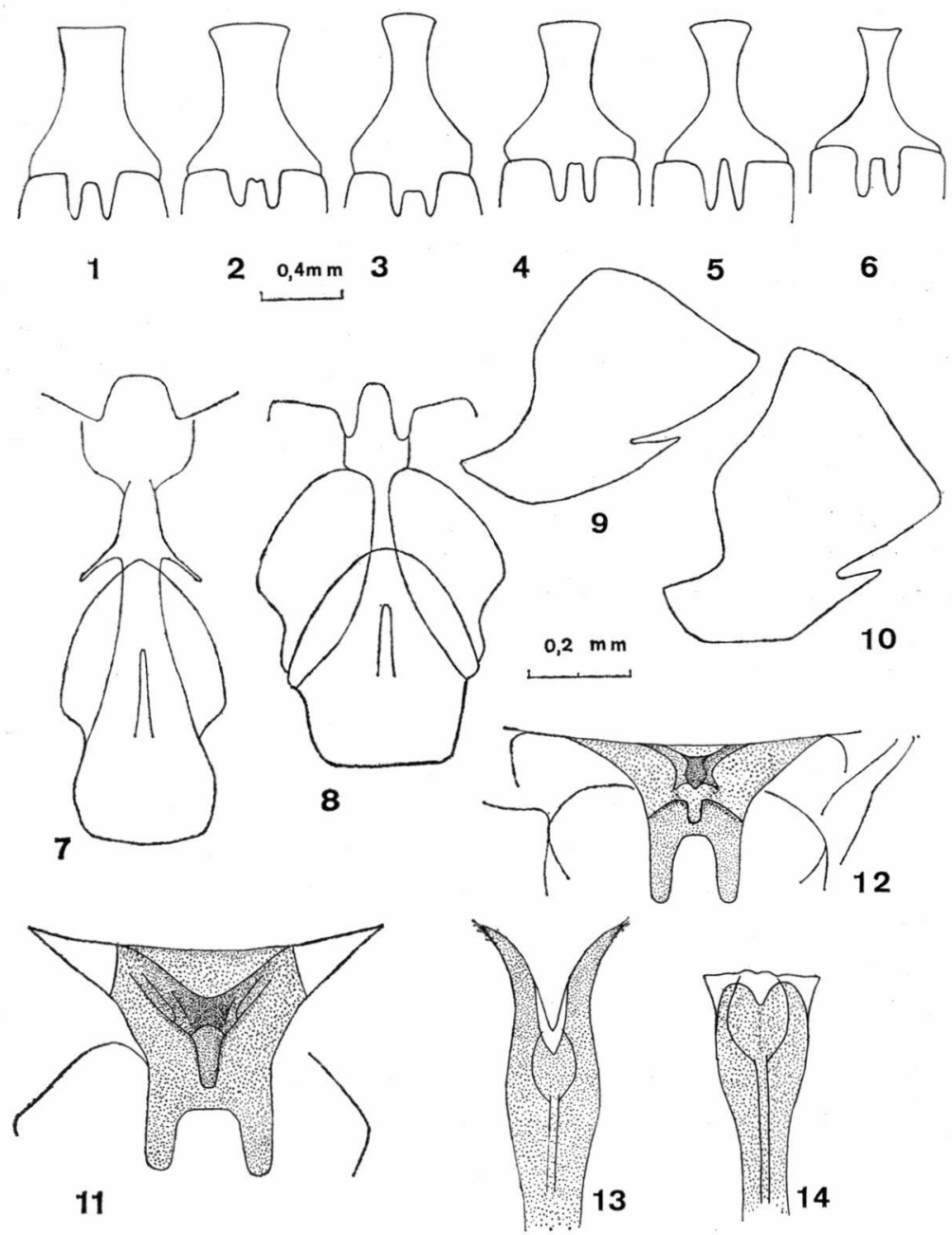

FIg. 1 à 6. - Différents aspects de la partie ventrale du segment IX et du lobe ventral de l'écaille vulvaire. $1,2,3$ : Potamophylax latipennis (C.) Neb.; 4, 5, 6 : Potamophylax cingulatus (Steph.).

FIG. 7 et 8 . - Appareil vaginal interne. 7 : Potamophylax latipennis (C.) NeB.; 8 : Potamophylax cingulatus (STEPH.).

Fig. 9 et 10. - Pièce centrale, vue de profil. 9 : Potamophylax latipennis (C.) Neb.; 10 : Potamophylax cingulatus (Steph.).

FIG. 11 et 12 . - Ouverture vaginale. 11 : Potamophylax latipennis (C.) NEB.; 12 : Potamophylax cingulatus (STEPH.).

Fig. 13 et 14 . - Pénis, vue ventrale. 13 : Potamophylax latipennis (C.) Neb.; 14 : Potamophylax cingulatus (STEPH.). 
- les pièces chitineuses entourant le vestibule vaginal sont nettement plus développées chez $P$. cingulatus et cette différence apparaissait déjà dans les dessins de D. E. Kimmıss [1944].

- la pièce centrale, vue de profil est haute et obtuse chez $P$. cingulatus (fig. 10), pointue chez $P$. latipennis (fig. 9).

c) Cependant, ces caractères internes ne peuvent être observés que sur des insectes disséqués et éclaircis à la potasse; cette étude est assez longue si l'on dispose d'un matériel abondant. C'est pourquoi il est plus aisé d'observer le vestibule par l'ouverture vaginale, les lobes mous étant écartés. Les dessins ainsi obtenus (fig. 11 et 12) montrent des différences permettant de caractériser plus rapidement les femelles des deux espèces. Chez $P$. latipennis (fig. 11) l'orifice situé à l'arrière du lobe médian, immédiatement au-dessus de ce lobe sur la figure, est bordé de part et d'autre par une limite chitinisée directement prolongée vers la partie supérieure. Chez $P$. cingulatus (fig. 12), cette limite, après un angle bien marqué de part et d'autre de l'orifice, est prolongée vers le bas, formant ainsi une gouttière qui n'apparaît pas chez $P$. latipennis. Cette différence correspond à la présence, chez $P$. cingulatus, de pièces annexes plus développées autour du vestibule vaginal. Elle s'est révélée constante sur le matériel pyrénéen étudié, comprenant 79 de $P$. cingulatus et $67 \%$ de $P$. latipennis.

d) En rapport avec cette structure des appareils génitaux des femelles, il est intéressant de noter des différences chez les $\hat{f}$, portant sur la morphologie du pénis. Ce dernier apparaît nettement bifide chez $P$. latipennis, avec deux pointes chitinisées, longues et divergentes - ce caractère n'existant pas chez $P$. cingulatus (fig. 13 et 14).

\section{2. - Remarques écologiques.}

Dans les régions où les deux espèces ont été récoltées, $P$. cingulatus a montré des exigences écologiques différentes de celles de $P$. latipennis. Ainsi D. E. Kimmins [1944] note, dans le district des lacs anglais, la présence de $P$. cingulatus dans les petits ruisseaux, celle de $P$. latipennis dans les grands ruisseaux, les rivières et le bord des lacs. L. Botosaneanu [1959], dans son étude des trichoptères du mont Banat trouve $P$. cingulatus dans les « torrents impétueux et froids de la forêt de conifères (eaux de type II) » et $P$. latipennis dans les « torrents hémisténothermes d'altitude moyenne et basse ainsi que dans les rivières de montagne (eaux de type VIII et IX) ». En Autriche, A. Adlmannseder [1965] situe $P$. cingulatus dans la région des sources, $P$. latipennis dans les ruisseaux et le cours supérieur de la rivière Antiesen. 


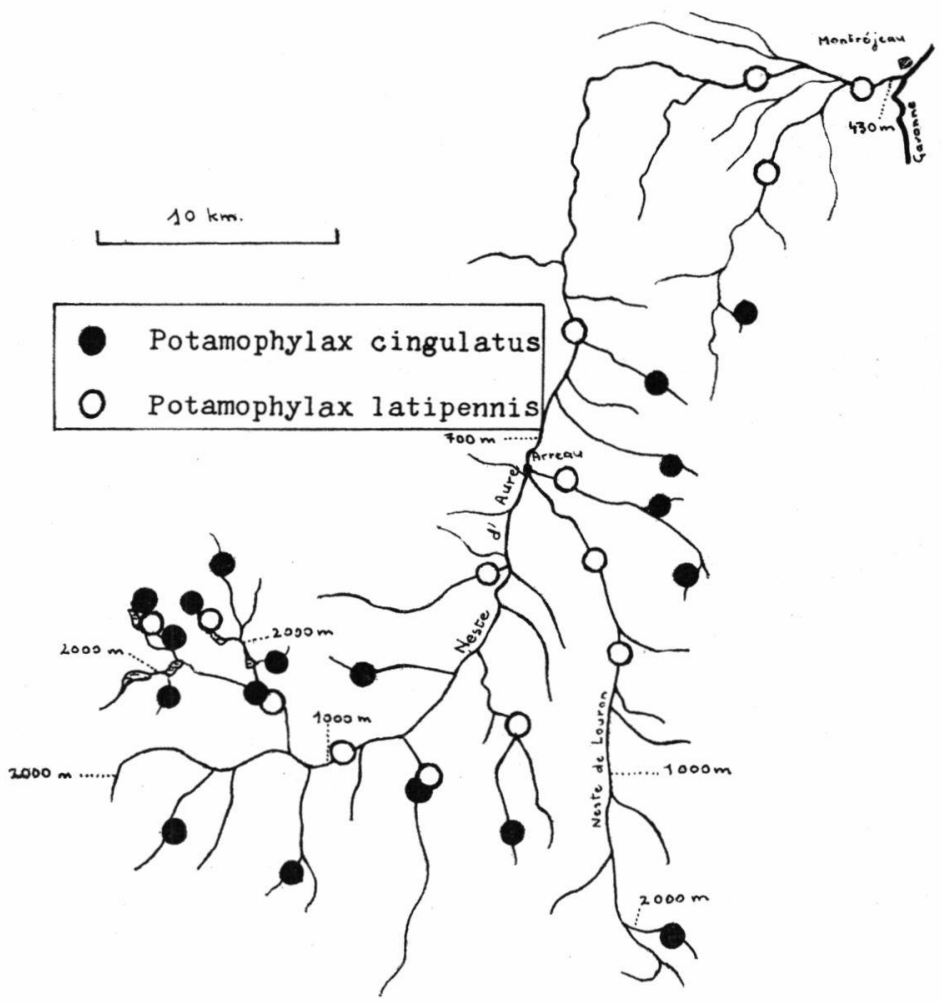

Carte : Répartition de Potamophylax latipennis (C.) Neb. et de Potamophylax cingulatus (STEPH.) dans la vallée d'Aure (Hautes-Pyrénées).

$P$. cingulatus apparaît donc comme plus sténotherme que $P$. latipennis. Il en est de même dans les Pyrénées centrales, avec pour la vallée de la Neste d'Aure, les particularités suivantes (voir carte) :

- dans la partie inférieure, entre 500 et $1000 \mathrm{~m}$ d'altitude, $P$. latipennis est fréquente dans la rivière et dans ses affluents, $P$. cingulatus est localisée au voisinage immédiat des sources dans les ruisseaux affluents.

- entre 1000 et $1800 \mathrm{~m}$, les deux espèces cohabitent dans les mêmes stations, en particulier vers $1300 \mathrm{~m}$ sur plusieurs affluents.

- au-dessus de $1800 \mathrm{~m}, P$. cingulatus fréquente les sources et les torrents froids issus des névés, $P$. latipennis la zone littorale des lacs subalpins. 


\title{
RESUME
}

Les différences de l'appareil $q$ interne de Polamophylax cingulatus et l'. latipennis sont visibles extérieurement, par l'ouverture vaginale (fig. 11 et 12) et permettent une distinction rapide des deux espèces. Jeurs caractères écologiques sont différents.

\author{
ON THE DETERMINATION OF THE FEMALES \\ OF POTAMOPHYLAX LATIPENNIS (CURT) NEBOISS \\ AND POTAMOPHYLAX CINGULATUS (STEPHENS)
}

The differences between the internal apparatus of the $q$ of $P$. cingulatus and $P$. latipennis may be seen externally (fig. 11 et 12) and allow a quick distinction of the two species.

The ecological characters are different.

\section{UEBER DIE BESTIMMUNG DER WEIBCHEN \\ DER POTAMOPHYLAX LATIPENNIS (CURT.) NEBOISS \\ UND DER POTAMOPHYLAX CINGULATUS (STEPHENS)}

Die Unterschiede der weiblichen inneren Organe der $P$. cingulatus und der P. latipennis sind äusserlich sichtbar (fig. 11 et 12) und erlauben eine schnelle Unterscheidung der beiden arten. Ihre oekologischen Merkmale sind verschieden.

\section{TRAVAUX GITÉS}

Adumannseder (A.). 1965. -..- Faunistisch-ökologische Untersuchungen im Flußgebiete der Antiesen unter besonderer Berucksichtigung der Trichopteren. Jahr. Oberösterr. Musealver., $100: 386-421$.

Botosaneanu (L.). 1959. - C Cercetari asupra Trichopterelor din Masivul Retezat si Muntii Banatului. Bibl. biol. anim. Bucarest, 1, 165 p.

Kimmins (D. E.). 1944. - Supplementary notes on the Trichoptera of the English I.ake District. Entomol., 7 \% : 81-83.

Maclachian (R.). 1874-1880. - A monographic Revision and Synopsis of the Trichoptera of the european Fauna. London, 523 p.

Mosely (M. E.). 1939. - The British Caddisflies (Trichoptera). A Collector's Handbook.London, 320 p.

Neborss (A.). 1963. — The Trichoptera Types of species described by J. Cuntis. Beitr. Ent., $13(5 / 6)$ : 582-635.

Saнmid (F.). 1955. - Contribution à l'étude des Limnophilidae (Trichoptera). Mitt. Schweiz. Entomol. Ges., 28, 245 p.

Tomaszewski (C.). 1962. - Bemerkungen über die Beschreibungen der Arten Potamophylax latipennis (CuRT.) und Hydropsyche angustipennis (Curt.). Ann. zool. Pologne, 20 (9) : 185-192.

(Station biologique du lac d'Orédon, Laboratoire de Zoologie de la Faculté des Sciences, Toulouse.) 